\title{
Preface: New language versions of MAIN: Multilingual Assessment Instrument for Narratives - Revised
}

\author{
Natalia Gagarina \\ Leibniz Zentrum Allgemeine Sprachwissenschaft
}

\section{Josefin Lindgren}

Leibniz Zentrum Allgemeine Sprachwissenschaft; TU Dortmund

\section{November 2019, Uppsala.}

At last. The Revised version of the Multilingual Assessment Instrument for Narratives (MAIN-Revised, part of the LITMUS ${ }^{1}$ battery) is about to be launched. The content has been polished up to the very end. The formatting is almost perfect. Over the past few years, ZAS Papers in Linguistics (ZASPiL) 63 with its Background on MAIN-Revised, how to use it and adapt it to other languages and 5 revised language versions (English, German, Russian, Swedish, and Turkish for the bilingual Turkish-speaking population in Sweden) has been prepared to be published. Now, Ute Bohnacker and Natalia Gagarina are sitting at the Linguistics Department, Uppsala University and are having long telephone talks with Christina Beckmann at the Leibniz-ZAS in Berlin, who is responsible for the technical part. It is not easy to organize the volume as we think it would be best: We want to publish the Introduction online with immediate access for everyone, but also provide links to the respective language versions of MAIN, so that prospective users first take note of the rules of use, agree to them, and then can enjoy the selected language version. Last-minute technical glitches need to be solved. But now we are there: Late at night on 26 November 2019, the volume finally sees the light of day and is online (https://zaspil.leibniz-zas.de/issue/view/53).

The next ZASPiL volume, number 64, has already been planned for some time and is currently in the making. Now that the Revised version of MAIN (2019) has been launched, further language versions are being worked on in every corner of the world. ZASPiL 64 will contain revised and new language versions of MAIN and should appear in early March 2020. We know

\footnotetext{
${ }^{1}$ LITMUS (Language Impairment Testing in Multilingual Settings) is a battery of tests that have been developed in connection with the COST Action IS0804 Language Impairment in a Multilingual Society: Linguistic Patterns and the Road to Assessment (2009-2013). Financial support by COST is hereby gratefully acknowledged.
} 
that this mammoth project will not be easy to realize, especially given the restricted resources that we have at our disposal, but we are driven by the common spirit of MAIN and its research network, by the desire of all contributors for this endeavour to see the light of day, and by a never-ending flow of researchers who are eager to contribute. And Josefin Lindgren has got a position at the Leibniz-ZAS in 2020 for several months to help Natalia finish the work.

\section{Mid-January 2020, Berlin.}

The coordination of the development of new and revised language versions of MAIN for ZASPiL 64 is continuing. A number of researchers express a wish to also contribute papers that describe the process of adapting MAIN to their respective language(s). Even though the final number of contributions for the volume has been set, new requests keep coming in and we have heated debates about whether to accept more new languages or not. Which language will we not accept? To whom we will first say: "It is too late" or "This is not good enough"? This is not an easy task. So, while we are writing these lines, we still do not know the exact number of language versions that are to be included in the volume, because we have decided to give the opportunity to everyone who has expressed a serious wish to adapt MAIN to their language(s).

\section{March 2020, Berlin.}

Since the beginning of March (actually even earlier) the world has changed. The Corona pandemic has struck. As of today, 27 March, when we are writing these lines, more than half a million people are infected with COVID-19 (according to the Johns Hopkins Institute in the US, 549,604, 11:49 AM; the Robert Koch Institute in Germany reports similar numbers). The numbers of infections are growing dramatically each day. It is not easy to concentrate, as people are dying, people are getting ill around us, life changes, and the universities and research institutes around the world are going into lock-down, but we must go on.

We now have to reconsider the timing of our initial publication plan. We decide to keep the main structure of the volume as originally planned but reconsider the 'internal' content. At the moment, we have around 70 new and revised language versions and around 25 chapters describing the adaptation processes, and we will start with them. Additional chapters and language versions will be added as soon as they are submitted.

For these working papers, each author is responsible for the content of their respective chapter. As editors of this ZASPiL volume, we are in contact with the authors during the process of adapting MAIN to a certain language and during their writing of the chapter. We check for general content and form and advise the authors how to present and shorten parts of their chapters that introduce the respective language and its cultural history. We also discuss contributors' questions about potential revisions of the pictorial stimuli for MAIN, e.g. for Asia, Africa and, specifically, Iran. Throughout, we point out the strict rules for adapting MAIN, so that the authors of new and/or revised language versions can ensure that all necessary requirements for high-quality adaptation have been met. We also make sure that all MAIN language versions have the same basic layout, not an easy task when languages are written 
using different writing systems (e.g. Arabic, Chinese, Cyrillic, Devanagari, Hebrew, Latin); Josefin does her best and communicates with the authors about all major discrepancies found. Some language versions are already done, many more to come. As volume editors we cannot be held responsible for the quality of the language adaptations; this responsibility lies with the authors of the individual language versions. The same also goes for the content of the chapters describing this process of adaptation. We make the decision to read all chapters, return them to the authors with comments and ask for revisions. ${ }^{2}$ This process will take several months, and as this could become a never-ending process, at some point we need to stop, but the chapters will not undergo double blind peer-review.

We have to specify that the chapters are not peer-reviewed.

\section{June 2020, Berlin and Groningen.}

We are now behind the deadline (again), new requests for language adaptations are still coming in, and the COVID-19 situation is not becoming any better. What to do? We realize that we will not manage to publish the volume before the summer break. We need to revise our plan. We set a new deadline for the chapters outlining the adaptation process - at this point, there are almost 30 chapters and some of them have been done for a while, but others still need revision. We also take the decision to reserve time for finalizing the new language versions till the end of 2020. The language versions in the volume will appear as links anyway, and these links will be activated one after the other, as new versions will be coming in. As things stand now, there should be between 70 and 80 languages. Wow, is this really going to happen?!

\section{August 2020, Berlin and Groningen.}

At last. All the chapters are there. The content has been worked on as much as possible. The formatting is almost perfect. ZASPiL 64 with the Preface, an Introductory chapter "MAIN Revised, how to use it and adapt it to other languages" and 33 chapters describing language adaptations have been prepared to be published. Josefin Lindgren has had long talks with Christina Beckmann and Nathalie Topaj, who are responsible for the technical parts. It is not easy to organize the volume as we think it would be best: We want to publish the Preface, the Introductory chapter, and all chapters describing the language versions online with immediate access, but also provide links to all 70 or 80 language versions of MAIN, so that everyone first takes note of the rules of use, agrees to them and then can enjoy the selected language version. But now we are there: finally, the volume sees the light of day and is online with 33 chapters. These chapters describe the processes of translating and adapting the Revised version of MAIN (2019) to languages belonging to different language families and spoken in various parts of the world (Arabic, Bulgarian, Cantonese (Chinese), Catalan, Croatian, Danish, Dutch, Estonian, (West) Frisian, Scottish-Gaelic, Gondi, Greek, Halbi, Hindi, Icelandic, Irish (Gaeilge), Italian, Kam, Kannada, Luxembourgisch, Malayalam, Mandarin (Chinese), Polish, (Brazilian)

\footnotetext{
${ }^{2}$ Some authors revise their papers only once, others many times (e.g. Uma Chimirala revised her chapter on Gondi, Halbi and Hindi by Chimirala an impressive 14 times).
} 
Portuguese, Serbian, Shughni, Slovak, Spanish, Tagalog/Filipino, Tajik, Torwali, Turkish, Urdu, Vietnamese, Yakut as well as chapters describing the use of MAIN in South Africa and with Norwegian-Russian bilinguals; MAIN-versions for these languages and many others are or will be available). Many of the chapters also give summaries of research that has been carried out with MAIN in the specific language(s) or present first results from pilot studies. Most chapters provide short descriptions of the grammar of the respective language(s) and/or detail the context in which they are spoken.

When you go through or read the volume, please note that for some of the MAIN language versions, there is no chapter on the adaptation process, e.g. Sangho. Note also that there are some chapters describing the process of language adaptation and some pilot results, but that no version of MAIN for this particular language is yet available. This is the case for e.g. Tajik and Shughni. MAIN versions for these languages do exist, however, although they have not been published as official versions, and the contact details of the authors can be found on the MAIN homepage, Subsection: 'Worldwide Network' (https://main.leibniz-zas.de/en/worldwide-

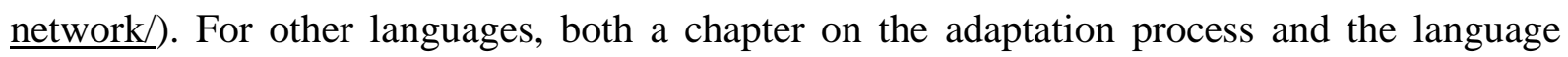
version are included in the present volume. So please carefully check the content.

Our work as editors of this volume has not always been easy, but we have managed it, hopefully not too badly. We are very happy to finally be able to publish this ZASPiL 64 volume and we would like to thank all the authors for having been our companions on this long and demanding, but very fruitful, journey.

\section{Natalia Gagarina and Josefin Lindgren \\ Berlin and Groningen}

P.S. The papers in this volume have not undergone a process of double-blind peer-review, and there is considerable variation in their quality. We want to emphasize that each author is responsible for the quality of the content of their respective paper. As editors we have read all contributions carefully and provided feedback and suggestions for improvements, both regarding the content and regarding coherence and clarity of the writing. The individual authors vouch for the quality of their MAIN language version described in the papers, as they were all required to follow the same rigorous criteria for translation and adaptation that were elaborated in Gagarina et al. (2012) and further revised in Bohnacker and Gagarina (2019). All existing language versions of MAIN-Revised can be accessed here (after registration). New language versions of MAIN will be added continuously throughout 2020, as they become ready. 